\title{
RELAÇÃO DO PERFIL EPIDEMIOLÓGICO DOS CUIDADORES DE IDOSOS COM DEMÊNCIA E A SOBRECARGA DO CUIDADO*
}

\author{
Bruna Silva Leite ${ }^{1}$, Alessandra Conceição Leite Funchal Camacho ${ }^{2}$, Maria Virginia Lyra Jacoud ${ }^{3}$, Mariana \\ Stavale de Almeida de Barros Santos ${ }^{3}$, Cintia Raquel da Costa de Assis ${ }^{4}$, Fabiana Lopes Joaquim ${ }^{5}$
}

\begin{abstract}
RESUMO: Objetivou-se avaliar o perfil sociodemográfico e clínico dos cuidadores e sua relação com a sobrecarga proveniente do cuidado ao idoso com demência. Estudo descritivo transversal, amostra não probabilística com 94 cuidadores de idosos com demência, realizado num Centro de Atenção à Saúde do Idoso no estado do Rio de Janeiro. A coleta de dados ocorreu de fevereiro a junho de 2016, por meio de questionário sociodemográfico e escala de Zarit. Quarenta e seis (50\%) cuidadores apresentaram sobrecarga moderada, 35 (38\%) pouca sobrecarga e $11(12 \%)$ sobrecarga moderada/severa; 71 (77\%) cuidadores usavam de 1 a 5 medicamentos e $20(22 \%) 6$ a 11 medicamentos. Observou-se as tendências: indivíduos com rendas mais baixas evidenciaram maiores níveis de sobrecarga; cuidadores com menor tempo de experiência possuíam sobrecarga moderada; quanto maior a sobrecarga, menor a média da idade. Verificou-se que a sobrecarga do cuidado coloca o cuidador em condições de vulnerabilidade biológica e psicológica.
\end{abstract}

DESCRITORES: Demência; Cuidadores; Enfermagem familiar; Idosos; Vulnerabilidade.

\section{RELATIONSHIP BETWEEN THE EPIDEMIOLOGICAL PROFILE OF CAREGIVERS OF ELDERLY PEOPLE WITH DEMENTIA AND OVERBURDEN OF CARE}

\begin{abstract}
The present study aimed to evaluate the sociodemographic and clinical profile of caregivers and their relationship with overburden of care of elderly people with dementia. Descriptive cross-sectional study, non-probability sample with 94 elderly people suffering with dementia, conducted at a Care Center for Elderly in the state of Rio de Janeiro. Data was collected from February to June 2016, through a questionnaire on sociodemographic aspects and Zarit Inventory. Forty-six (50\%) caregivers had moderate overburden, 35 (38\%) had little overburden and 11 (12\%) moderate/severe overburden; 71 (77\%) caregivers took 1 to 5 drugs and $20(2 \%)$ took 6 to 11 drugs. The following trends were observed: individuals with lower income experienced higher levels of overburden; less experienced caregivers had moderate overburden; the greater the overburden of caregivers, the lower the average age of these individuals. Also, overburdened caregivers were in conditions of biological and psychological vulnerability. DESCRIPTORS: Dementia; Caregivers; Family nursing; Elderly; Vulnerability.
\end{abstract}

\section{ASOCIACIÓN ENTRE EL PERFIL EPIDEMIOLÓGICO DE LOS CUIDADORES DE ANCIANOS CON DEMENCIA Y LA SOBRECARGA DEL CUIDADO}

RESUMEN: Fue objetivo del estudio evaluar el perfil sociodemográfico y clínico de los cuidadores y su relación con la sobrecarga del cuidado al anciano con demencia. Estudio descriptivo transversal, con muestra probabilística con 94 cuidadores de ancianos con demencia, realizado en un Centro de Atención a la Salud del Anciano en estado de Rio de Janeiro. Los datos fueron obtenidos de febrero a junio de 2016, por medio de cuestionario sociodemográfico y escala de Zarit. Cuarenta y seis (50\%) cuidadores presentaron sobrecarga moderada, 35 (38\%) poca sobrecarga y $11(12 \%)$ sobrecarga moderada/severa; $71(77 \%)$ cuidadores usaban de 1 a 5 medicamentos e 20 (22\%) 6 a 11 medicamentos. Se observaron las siguientes tendencias: individuos con rentas más bajas evidenciaron mayores niveles de sobrecarga; cuidadores con menos tiempo de experiencia tenían sobrecarga moderada; cuanto mayor la sobrecarga, menor la media de edad. Se verificó que la sobrecarga del cuidado pone el cuidador en condiciones de vulnerabilidad biológica y psicológica.

DESCRIPTORES: Demencia; Cuidadores; Enfermería familiar; Ancianos; Vulnerabilidad.

\footnotetext{
*Artigo extraído da dissertação de mestrado intitulada: "A vulnerabilidade dos cuidadores de idosos com demência: estudo descritivo transversal". Universidade Federal Fluminense, 2016.

${ }^{1}$ Enfermeira. Mestre em Ciências do Cuidado em Saúde. Universidade Federal Fluminense. Japeri, RJ, Brasil.

${ }^{2}$ Enfermeira. Doutora em Enfermagem. Docente de Enfermagem da Universidade Federal Fluminense. Niterói, RJ, Brasil.

${ }^{3}$ Discente de Enfermagem. Universidade Federal Fluminense. Niterói, RJ, Brasil.

${ }^{4}$ Enfermeira. Mestranda em Ciências do Cuidado em Saúde. Universidade Federal Fluminense. Niterói, RJ, Brasil.

${ }^{5}$ Enfermeira. Doutoranda em Enfermagem. Universidade Federal Fluminense. Niterói, RJ, Brasil.
} 


\section{INTRODUÇÃO}

Os resultados atuais do último censo evidenciam a diminuição no índice do crescimento populacional e o aumento no número de idosos, que já representam cerca de $11 \%$ da população. Com a estimativa de vida da população mais levada, nota-se significativo aumento de doenças crônico-degenerativas relacionadas à idade. Dentre essas, as demências, e a Doença de Alzheimer (DA) se destaca como a mais corriqueira ${ }^{(1)}$.

Nessa perspectiva, o envelhecimento pode ser definido como a diminuição gradativa da capacidade funcional, a qual ocorre no período posterior à maturidade reprodutiva. $O$ esquecimento, com o desenvolver da velhice, é constante queixa dos indivíduos. Esse transtorno de memória, relacionado à idade, tem grande incidência nos idosos em geral e deve ser observado de modo a diferenciar o normal do patológico ${ }^{(2)}$.

Com o aumento na estimativa de vida da população, observa-se, em concomitância, significativo aumento nos casos de demências. A demência é considerada uma síndrome essencialmente de natureza crônica e progressiva, causada por vários déficits cerebrais que afetam a memória, a capacidade de pensamento, o comportamento, assim como a capacidade de realizar atividades de vida diária. No ano de 2010, estimou-se que 35,6 milhões de pessoas foram diagnosticadas com demência. Anualmente são identificados 7,7 milhões de novos casos, o que significa que, a cada quatro segundos, em algum lugar do mundo, é detectado um novo caso de demência ${ }^{(3)}$.

Devido ao comprometimento cognitivo do idoso em processo demencial, faz-se necessária a presença de cuidador para auxiliar na realização das atividades de vida diárias. O cuidador está presente na rotina desse idoso, presenciando todas as manifestações da demência e com o encargo de zelar pela integridade física do mesmo. Logo, fica exposto às mudanças comportamentais que podem ser apresentadas pela pessoa idosa e precisa ter o manejo adequado de suas ações, para conduzir aquela circunstância de forma a evitar prejuízos à sua saúde e à do idoso ${ }^{(4)}$.

O cuidado ao idoso com demência ocasiona inúmeras e distintas implicações à vida dos cuidadores. Esses indivíduos sofrem com alterações no sono, afeto, humor e depressão, fatores que repercutem no aspecto físico, emocional, psicológico e social. O progressivo aumento da dependência dos idosos acarreta o agravo na saúde dos cuidadores ${ }^{(5)}$.

Dessa forma, o transtorno demencial torna-se via de mão dupla, na qual a demência gera impactos na qualidade de vida do cuidador e sua qualidade de vida pode influenciar na prestação dos cuidados. Portanto, tanto o cuidador quanto o idoso necessitam de atenção especializada e focada em suas necessidades.

Esse fato permite o planejamento de cuidados direcionados às necessidades desse público, haja vista que a manutenção da qualidade de vida do cuidador, além de trazer benefícios para si, atua na melhoria da qualidade dos cuidados prestados. Portanto, o objetivo deste estudo foi de descrever o perfil sociodemográfico e clínico dos cuidadores e avaliar sua relação com a sobrecarga proveniente do cuidado ao idoso com demência.

\section{- MÉTODO}

Estudo de abordagem quantitativa do tipo descritiva transversal. O local do estudo foi o Centro de Atenção à Saúde do Idoso e seus Cuidadores (CASIC), unidade ambulatorial geriátrica de assistência ao idoso da Universidade Federal Fluminense localizada no município de Niterói, Rio de Janeiro. A coleta de dados ocorreu de fevereiro a junho de 2016 durante a consulta de enfermagem

O estudo contou com 92 participantes, sendo a amostra não probabilística. Constituíram critérios de inclusão no estudo: ser cuidador informal de idosos portadores de demência; frequentar o CASIC; ser o cuidador principal por no mínimo seis meses. Os critérios de exclusão foram: não ter experiência prática como cuidador de idosos com demência; presença de perturbação da comunicação que dificultasse a realização dos testes; faltar a três consultas consecutivas sem justificativa prévia. 
O protocolo adotado para a coleta de dados com os cuidadores de idosos com demência foi a entrevista estruturada, realizada durante a consulta de enfermagem.

Para a coleta de dados foram elencados dois instrumentos: o questionário sociodemográfico criado para a pesquisa, com o objetivo de identificar o perfil do cuidador de idosos com demência através das variáveis clínicas e sociodemográficas, e a escala de Zarit para mensurar a sobrecarga proveniente do cuidado.

A Escala Burden Interview é uma das escalas utilizadas principalmente para a avaliação de sobrecarga em cuidadores de pacientes idosos com demência. A validação da escala foi feita em 2002, através de um estudo realizado no Hospital das Clínicas da Faculdade de Medicina da Universidade de São Paulo (Ipq-HCFMUSP) e no Centro de Atenção Integrada Saúde Mental da Santa Casa de Misericórdia, também em São Paulo(6).

Esse inventário possui 22 questões. As respostas podem ser: nunca (0 pontos), raramente (1 ponto), algumas vezes ( 2 pontos), frequentemente ( 3 pontos) e sempre (4 pontos). A soma total dos itens pode variar de 0 - 88 pontos. Quanto maior a pontuação, maior é a sobrecarga.

O nível de sobrecarga dos cuidadores é qualificado com os seguintes escores: $<21=$ pouca sobrecarga; entre 21 e 40 = sobrecarga moderada; entre 41 a $60=$ sobrecarga moderada a severa e entre 61 a 88 = sobrecarga severa ${ }^{(7)}$.

A análise dos resultados envolveu a construção de planilha eletrônica no programa Exce ${ }^{\circledR}$, em dupla digitação e validados para a comparação das digitações. Após a validação e interpretação dos dados, eles foram descritos utilizando gráficos e tabelas. Para analisar a normalidade dos dados, foi aplicado o teste Kolmogorov-Smirnov, já que a amostra possui mais que 50 participantes. Esse teste é importante para determinar a escolha correta dos próximos testes de avaliação da associação dos dados. A distribuição normal significa que eventos aleatórios seguem um padrão. Já a não normalidade demonstra que alguma das variáveis que descrevem um acontecimento evidencia distribuição de probabilidade que não seja a normal, por razões inerentes ao fenômeno.

A medida de tendência central utilizada para todos os dados com distribuição não normal foi à mediana, já a de dispersão o intervalo interquartílico. Exceto para idade e tempo como cuidador, pois apresentaram distribuição normal e, portanto foi atribuída média e desvio padrão.

Os Testes utilizados foram: ANOVA one-way e o Kruskal-Wallis. A análise de Variância ou ANOVA é uma eficaz técnica estatística desenvolvida por Fisher. Esse teste decompõe em vários elementos a variação entre os valores obtidos. O modelo mais simples desse teste é o que analisa os dados de um delineamento completamente casualizado ou ANOVA a um critério de classificação (ANOVA one way). Para que os resultados sejam válidos, é necessário que os dados apresentem distribuiç̧ão normal ${ }^{(8)}$.

Quando os dados apresentam distribuição não normal, não é recomendado confiar em uma análise de variância tradicional. Nesses casos, o Teste Kruskal-Wallis é uma alternativa e tem por finalidade comparar a medida de tendência central dos dados ${ }^{(8)}$.

A fim de calcular o poder de associação dos dados com distribuição normal, em que foi empregado o ANOVA, utilizou-se o programa estatístico $G^{*}$ Power 3.1.3. Já para verificar o tamanho do efeito de associação entre as variáveis com distribuição não normal, adotou-se o V de Cramer.

A pesquisa foi aprovada pelo Comitê de Ética e Pesquisa do Hospital Universitário Antônio Pedro (HUAP), sob o parecer $\mathrm{n}^{\circ} 1.220 .297$.

\section{RESULTADOS}

A Tabela 1 fornece o perfil sociodemográfico da amostra, destaque para a variável sexo, com 57 (62\%) feminino, idade entre 48 e 57 anos e estado civil casados para 48 (52,17\%). 
Tabela 1 - Dados sociodemográficos da amostra. Niterói, RJ, Brasil, 2016

\begin{tabular}{|c|c|c|c|}
\hline Variável & Categorias & $f(n)$ & fr $(\%)$ \\
\hline \multirow[t]{2}{*}{ Sexo } & Feminino & 57 & 62 \\
\hline & Masculino & 35 & 38 \\
\hline \multirow[t]{8}{*}{ Idade (anos) } & 18 a 27 & 2 & 2 \\
\hline & 28 a 37 & 1 & 1 \\
\hline & 38 a 47 & 8 & 8,7 \\
\hline & 48 a 57 & 30 & 32,6 \\
\hline & 58 a 67 & 29 & 32 \\
\hline & 68 a 77 & 13 & 14 \\
\hline & 78 a 87 & 8 & 8,7 \\
\hline & 88 a 97 & 1 & 1 \\
\hline \multirow[t]{3}{*}{ Escolaridade (anos) } & 0 a 9 & 26 & 29 \\
\hline & 10 a 19 & 63 & 68 \\
\hline & 19 a 20 & 3 & 3 \\
\hline \multirow[t]{5}{*}{ Estado Civil } & Casado & 48 & 52,17 \\
\hline & Viúvo & 6 & 6,52 \\
\hline & Separado/Divorciado & 17 & 18,47 \\
\hline & Solteiro & 12 & 13,04 \\
\hline & Outro concubinato & 9 & 9,8 \\
\hline \multirow[t]{5}{*}{ Renda } & $\mathrm{R} \$ 800,00$ a $\mathrm{R} \$ 3.000,00$ & 39 & 42 \\
\hline & $\mathrm{R} \$ 3.001,00$ a $\mathrm{R} \$ 6.000,00$ & 29 & 32 \\
\hline & $\mathrm{R} \$ 6.000,00$ a $\mathrm{R} \$ 12.000,00$ & 14 & 15 \\
\hline & $\mathrm{R} \$ 12.001,00$ a $\mathrm{R} \$ 24.000,00$ & 9 & 10 \\
\hline & $\mathrm{R} \$ 24.000,00$ a $\mathrm{R} \$ 34.000,00$ & 1 & 1 \\
\hline \multirow[t]{5}{*}{ Parentesco } & Cônjuge & 48 & 52 \\
\hline & Filho(a) & 6 & 6,5 \\
\hline & Nora/Genro & 17 & 18,5 \\
\hline & Irmão(a) & 13 & 13 \\
\hline & Outro parentesco & 9 & 10 \\
\hline \multirow[t]{2}{*}{ Trabalha } & Sim & 36 & 39 \\
\hline & Não & 56 & 61 \\
\hline
\end{tabular}

Foram identificados (Tabela 2) outros percentuais: Hipercolesteremia representou cinco (5,4\%) da amostra, dois $(2,2 \%)$ dos participantes do estudo relatam os seguintes problemas de saúde: Parkinson, Herpes Zoster, Nódulos na Tireoide, Gastrite, Depressão e Fibromialgia. As enfermidades menos frequentes, que ao menos um (1\%) dos integrantes da pesquisa tinham, foram: Transtorno de Ansiedade, Isquemia, Labirintite, Doença de Alzheimer, Prostatite, Cálculo renal e Transtorno Obsessivo Compulsivo. Vale ressaltar que na variável enfermidades, a Tabela 2 destaca a frequência em que essas apareceram na população estudada, sendo possível que um mesmo cuidador apresentasse várias patologias. 
Tabela 2 - Dados clínicos da amostra. Niterói, RJ, Brasil, 2016

\begin{tabular}{llcc} 
Variável & Categorias & $\mathbf{f}(\mathbf{n})$ & $\mathbf{f r}(\mathbf{\%})$ \\
\hline Enfermidades & Hipertensão Arterial Sistêmica & 43 & 46,7 \\
\cline { 2 - 4 } & Artrose & 39 & 42,4 \\
\cline { 2 - 4 } & Problemas na coluna & 36 & 39,1 \\
\cline { 2 - 4 } & Diabetes Mellitus & 14 & 15,2 \\
\cline { 2 - 4 } & Cardiopatia & 12 & 13 \\
\cline { 2 - 4 } & Osteoporose & 11 & 12 \\
\cline { 2 - 4 } & Hipotireoidismo & 10 & 10,8 \\
\cline { 2 - 4 } & Deficiência de vitaminas & 24 & 8,6 \\
\cline { 2 - 4 } & Outros & 1 & 25,6 \\
\hline \multirow{2}{*}{$\begin{array}{l}\text { Uso de Medicamentos } \\
\text { quantidade de medicamentos por dia) }\end{array}$} & Não usa nenhum medicamento & 71 & 77 \\
\cline { 2 - 4 } & 1 a 5 & 20 & 22
\end{tabular}

A Tabela 3 apresenta a análise descritiva das variáveis relacionadas ao cuidado ao idoso. Em relação ao tempo como cuidador, em meses, existe uma grande diversidade de intervalos. O tempo mínimo foi de seis meses e a partir desse valor foram criados intervalos semestrais para melhor demonstração.

Tabela 3 - Variáveis relacionadas ao cuidado. Niterói, RJ, Brasil, 2016 (continua)

\begin{tabular}{|c|c|c|c|}
\hline Variável & Intervalos & $f(n)$ & fr (\%) \\
\hline \multirow{18}{*}{ Horas gastas por semana } & 0 a 12 & 4 & 4 \\
\hline & 13 a 24 & 7 & 8 \\
\hline & 25 a 36 & 12 & 13 \\
\hline & 37 a 48 & 8 & 9 \\
\hline & 49 a 60 & 13 & 14 \\
\hline & 61 a 72 & 9 & 10 \\
\hline & 73 a 84 & 22 & 24 \\
\hline & 85 a 96 & 2 & 2 \\
\hline & 97 a 108 & 5 & 5,5 \\
\hline & 109 a 120 & 5 & 5,5 \\
\hline & 121 a 132 & 0 & 0 \\
\hline & 133 a 144 & 0 & 0 \\
\hline & 145 a 156 & 1 & 1 \\
\hline & 157 a 168 & 4 & 4 \\
\hline & 6 & 4 & 4 \\
\hline & 7 a 12 & 4 & 4 \\
\hline & 13 a 18 & 3 & 3 \\
\hline & 19 a 24 & 2 & 2 \\
\hline
\end{tabular}




\begin{tabular}{|c|c|c|c|}
\hline \multirow[t]{15}{*}{ Tempo como cuidador em meses } & 25 a 30 & 0 & 0 \\
\hline & 31 a 36 & 8 & 9 \\
\hline & 37 a 42 & 0 & 0 \\
\hline & 43 a 48 & 5 & 5,5 \\
\hline & 49 a 54 & 0 & 0 \\
\hline & 55 a 60 & 11 & 12 \\
\hline & 61 a 66 & 0 & 0 \\
\hline & 67 a 72 & 0 & 0 \\
\hline & 73 a 78 & 0 & 0 \\
\hline & 79 a 84 & 9 & 10 \\
\hline & 85 a 90 & 1 & 1 \\
\hline & 91 a 96 & 8 & 9 \\
\hline & 97 a 102 & 0 & 0 \\
\hline & 103 a 108 & 3 & 3 \\
\hline & $\geq 109$ & 30 & 33 \\
\hline
\end{tabular}

Ainda acerca das variáveis referentes ao cuidado, outro aspecto identificado foi relativo à divisão do cuidado ao idoso com outra pessoa. Dentre os cuidadores, $88 \%(n=81)$ dividia o cuidado com outro membro da família e 12\% $(n=11)$ realizava o cuidado integral sem nenhum tipo de ajuda.

Na Tabela 4, visualiza-se o teste de normalidade da população do estudo. Todos os dados apresentaram distribuição não normal, exceto a variável idade com p=0,169.

Tabela 4 - Teste de normalidade Kolmogorov-Smirnov. Niterói, RJ, Brasil, 2016

\begin{tabular}{lccc} 
& \multicolumn{3}{c}{ Kolmogorov-Smirnov } \\
\cline { 2 - 4 } & Estatística & DF & Sig. \\
\hline Idade & 0,082 & 92 & 0,169 \\
\hline Uso medicamentos & 0,246 & 92 & 0 \\
\hline Horas cuidado semana & 0,135 & 92 & 0 \\
\hline Tempo cuidador & 0,123 & 92 & 0,002 \\
\hline Divide cuidado & 0,504 & 92 & 0 \\
\hline Renda & 0,195 & 92 & 0
\end{tabular}

No que se refere à aplicação da escala de Zarit, 50\% (n=46) dos cuidadores apresentaram sobrecarga moderada, 38\% $(n=35)$ apresentavam pouca sobrecarga e 12\% $(n=11)$ apresentaram sobrecarga moderada/severa.

Relacionado à idade, foi aplicado o teste ANOVA para verificar a possível associação entre a idade e os resultados da Escala de Zarit. O resultado demonstrou que não houve diferença significativamente estatística entre essas variáveis, $p=0,402$. O nível de significância adotado foi $p \leq 0,05$.

Apesar de o valor de p não ter apresentado diferença significativamente estatística, ao considerar as médias dos cuidadores com pouca, moderada e moderada a severa sobrecarga, percebe-se, por intermédio da análise do poder de associação, que os dados apresentam uma tendência moderada ao seguinte resultado: quanto maior a sobrecarga menor a média da idade. Indivíduos com pouca sobrecarga tiveram a média de idade 61,17, com sobrecarga moderada 59,74 e como sobrecarga moderada/severa 55,36. 
No que concerne ao tempo como cuidador, o teste ANOVA a um fator constatou $p=0,091$. Isso demonstra que não houve diferença significativa entre os resultados da escala de Zarit e essa variável. O nível de significância adotado foi $\mathrm{p} \leq 0,05$. O poder de associação dessas variáveis foi de 0,508 e ao considerar as médias dos indivíduos com pouca (média: 97,34, desvio padrão: 62,5), moderada (média: 75,17, desvio padrão: 48,3) e moderada a severa sobrecarga (média: 106,36, desvio padrão: 44,9), é possível destacar que a menor média de tempo como cuidador foi nos cuidadores com sobrecarga moderada e a maior nos com sobrecarga moderada a severa. Assim, cuidadores com menos tempo de experiência apresentaram sobrecarga moderada.

No que diz respeito à renda, quando relacionada à escala de Zarit, por intermédio do teste Kruskal Wallis, não apresentou diferença significativamente estatística entre os dados, $p=0,224$. Contudo, a mediana da renda dos participantes com pouca sobrecarga foi $\mathrm{R} \$ 5.000,00$, e a amplitude interquartil foi de $\mathrm{R} \$ 4.612,00$. Esse fato demonstra que esse valor se deve a três participantes que estão fora do esperado nesse grupo.

Em seguimento, a mediana dos indivíduos com sobrecarga moderada foi $\mathrm{R} \$ 2.994$ e a amplitude interquartil foi de $\mathrm{R} \$ 4.777,00$; nos cuidadores com sobrecarga moderada a severa a mediana foi $\mathrm{R} \$ 2.200,00$ e a amplitude interquartil foi de $\mathrm{R} \$ 3.190,00$. O teste $V$ de Cramer mostrou associação grande entre essas variáveis, com um poder de 0,835 para o fato de que indivíduos com rendas mais baixas apresentam maiores níveis de sobrecarga.

A heterogeneidade dos dados pode ter sido um possível fator que explica a amostra não ter apresentado diferença significativamente estatística entre a análise das variáveis.

\section{- DISCUSSÃO}

Referente ao sexo, $62 \%(n=57)$ dos participantes era do sexo feminino. Os achados desse estudo corroboram com a literatura no que diz respeito ao fato da mulher ainda exercer, majoritariamente, o cuidado. Na sociedade brasileira, tradicionalmente, devido à cultura patriarcal e ao machismo, a mulher desempenha o papel de cuidadora familiar na figura da mãe e da dona de casa ${ }^{(9)}$.

Entretanto, observa-se que esse perfil está sendo modificado. Em outras pesquisas, nota-se que as mulheres representavam percentuais acima de $70 \%$. Esse fato também se evidenciou em outras investigações científicas ${ }^{(9-10)}$ que apresentaram percentuais entre 70,6\% e 87,6\%. Logo, o fato do cuidado ser exercido por homens em 38\% ( $n=35)$ da amostra, evidencia que esses indivíduos estão participando mais do cuidado e assumindo o papel de cuidadores principais.

Em relação à idade, $64,6 \%$ dos participantes estão na faixa de 48 a 57 anos e 58 a 67 anos. Destaca-se que as faixas etárias acima de 60 anos tem um percentual expressivo (58 a 67 com 32\%; 68 a 77 com 14\%; 78 a 87 com 8,7\% e 88 a 97 com 1\%), compondo mais de $50 \%$ da amostra.

Os dados mostram que idosos estão exercendo o papel de cuidador de outros idosos com demência. Esse achado corrobora com o observado em outras pesquisas que também evidenciaram mais de $40 \%$ da amostra de cuidadores com mais de 60 anos $^{(11)}$. Ou seja, os profissionais de saúde, além de atentarem para as necessidades do indivíduo como cuidador, precisam considerar as especificidades da faixa etária na qual se encontram.

Quanto à escolaridade, $71 \%$ possuía mais de 10 anos de estudo. Esse resultado não está de acordo com os achados de outras pesquisas ${ }^{(11-12)}$ nas quais a maior parte dos cuidadores tinham no máximo o ensino primário.

O grau de escolaridade, tanto do cuidador quanto do idoso, é uma informação muito relevante para a equipe de enfermagem. É a partir dela que o enfermeiro deve preparar o conteúdo das ações educativas e optar pela abordagem mais acessível para que, dessa forma, os resultados esperados possam ser alcançados ${ }^{(12)}$.

No que concerne ao estado civil, a maior parte dos integrantes da pesquisa eram casados, 52,17\%. Esse dado condiz com o encontrado em outras investigações com cuidadores. Pesquisas ${ }^{(11,13)}$ evidenciam percentuais entre $50,7 \%$ e $78,3 \%$ de indivíduos casados. 
A faixa da renda mensal dos cuidadores que apresentou um percentual mais elevado foi a de $\mathrm{R} \$ 800,00$ a $\mathrm{R} \$ 3.000,00$ com $42 \%$ e a de $\mathrm{R} \$ 3.001,00$ a $\mathrm{R} \$ 6.000,00$ com $32 \%$. Um estudo ${ }^{(9)}$ evidenciou que a renda média domiciliar girava em torno de $\mathrm{R} \$ 630$ reais, valor bem abaixo do encontrado no presente estudo.

Relacionado ao grau de parentesco, $52 \%(n=48)$ dos cuidadores eram cônjuges; $6,5 \%$ ( $n=6)$ filhos; nora ou genro $18,3 \%(n=17)$ e irmão(a) $13 \%(n=13)$ e outro tipo de parentesco. O fato de mais de $50 \%$ da população ser constituída por cônjuges vai ao encontro do resultado de pesquisas anteriores, as quais revelam que a família é a principal provedora de assistência ao idoso com demência. As mulheres, na maior parte dos casos, assumem a responsabilidade dos cuidados de forma mais intensa e prolongada ${ }^{(14)}$.

Entretanto, os achados relacionados às noras e aos genros contrastam com o que é encontrado em outras investigações. Na maioria das vezes, os filhos(as) ocupam o primeiro ou o segundo lugar como cuidadores. Esse quadro foi identificado em outros estudos ${ }^{(13,15)}$.

Referente ao vínculo empregatício, 61\% $(n=56)$ dos cuidadores não trabalhavam e 39\% ( $n=36)$ trabalhavam. Autores afirmam que os cuidadores podem não exercer nenhuma outra função remunerada pela falta de opção, uma vez que os idosos com demência necessitam de constante supervisão, o que intensifica a jornada de trabalho. Como não existe outra pessoa para assumir essa responsabilidade, o indivíduo acaba se desvinculando do trabalho e dedicando-se exclusivamente ao exercício do cuidado ao idoso com demência. Esta decisão pode acarretar em um desgaste psicológico ${ }^{(9)}$.

Os problemas de saúde enfrentados pelos cuidadores preocupam, pois eles são responsáveis pelo cuidado e, na maioria do tempo, ficam sozinhos em casa com os idosos. As enfermidades mais frequentes entre os cuidadores foram: Hipertensão Arterial Sistêmica (HAS) 46,7\%, Artrose 42,4\%, Problemas na coluna 39,1\%, Diabetes Mellitus 15,2\%, Cardiopatia 13\%, Osteoporose 12\%, Hipotireoidismo 10,8\%, Deficiência de vitaminas $8,6 \%$. As doenças dos cuidadores constituem um grande desafio para os profissionais de saúde. É importante destacar que alguns cuidadores já se encontram em idade avançada e há real necessidade da colaboração de outras pessoas para manter o cuidado ${ }^{(11)}$.

Um estudo observacional transversal realizado em Brasília encontrou a Hipertensão Arterial Sistêmica (HAS) como a doença mais frequente nos cuidadores. Entretanto, afirmam que esse achado já era esperado devido a grande parte desses indivíduos terem mais de 60 anos de idade. A HAS é uma doença crônica esperada para essa faixa etária. Outro aspecto relevante foi a queixa de dor, sendo mais citadas na coluna ou $\operatorname{costas}^{(9)}$. Esse dado está de acordo com o que foi encontrado na presente pesquisa.

A queixa de dor física foi identificada em outra investigação ${ }^{(15)}$ como o problema mais prevalente nos cuidadores. Foi observada, também, tendência a pior autopercepção de saúde física entre os cuidadores de pacientes com Doença de Alzheimer, quando comparados a outros tipos de demência.

Ressalta-se que 1\% da amostra não usa nenhum medicamento, enquanto $77 \%$ usam de 1 a 5 medicamentos e $22 \%$ de 6 a 11 medicamentos. A polifarmácia utilizada pelos cuidadores está relacionada ao aumento do risco e da gravidade das reações adversas aos medicamentos, precipitar interações medicamentosas, ocasionar toxicidade cumulativa, provocar erros de medicação, além de reduzir a adesão ao tratamento e elevar a morbimortalidade ${ }^{(16)}$.

Pesquisas evidenciam ${ }^{(9-10)}$ que o elevado uso de medicamentos por cuidadores é uma prática comum, sendo na maioria das vezes utilizado sem prescrição médica, para alívio de dores e resolução de problemas com insônia.

Na população do estudo, o intervalo de horas disponibilizadas para o cuidado mais frequente foi alto, entre 73 a 84 horas por semana, cerca de 10 a 12 horas diárias. Ainda que o cuidador tenha uma segunda pessoa encarregada pelo cuidado, o mesmo continua a ocupar seu pensamento com o desempenho das tarefas diárias com o idoso. Alguns cuidadores não conseguem se desvincular totalmente do cuidado, embora não estejam diretamente responsáveis por ele.

As repercussões na vida do cuidador são evidentes, a demanda de tempo requerida pelo cuidado faz com que a vida do cuidador não receba a atenção necessária ${ }^{(16)}$. Esse fato pode influenciar o cuidado ao idoso. A equipe de saúde deve estar atenta às necessidades psicobiológicas, condições de vida e de 
saúde do idoso a fim de promover o cuidado de ambos ${ }^{(17)}$.

Em relação à divisão do cuidado, a análise da estatística descritiva simples mostra que entre os indivíduos que não dividiam o cuidado o índice de sobrecarga foi maior que $80 \%$. Esse achado vai ao encontro do que é observado na literatura ${ }^{(11)}$. A divisão do cuidado com o cuidador secundário pode contribuir para um quadro de menor sobrecarga.

É comum observar casos de sobrecarga nos cuidadores. As tarefas realizadas por eles tendem a ser assumidas por uma única pessoa, denominada de cuidador principal. Logo, centraliza-se, nesse indivíduo, o elo entre idoso, família e a equipe de saúde.

Eles assumem e se responsabilizam pelas ações do cuidado, incluindo as atividades de higiene, alimentação, supervisão domiciliar, acompanhamento nos serviços de saúde, condução da terapia medicamentosa, apoio na prática de exercícios físicos, entre outras, que surgirem em função da dependência e necessidades de saúde do idoso com demência. Esse fato acarreta total comprometimento da rotina do responsável pelo cuidado, o que pode contribuir para a sua sobrecarga ${ }^{(10)}$.

Outro aspecto a ponderar refere-se à menor média da variável tempo como cuidador foi encontrada em participantes que tinham sobrecarga moderada e a maior nos com sobrecarga moderada a severa. Um estudo ${ }^{(11)}$ realizado em 2012, identificou que os cuidadores com mais de 24 meses de experiência relataram que as atividades foram se tornando desgastantes. Enfatizaram, ainda, o abalo do seu estado emocional, junto ao cansaço físico. Além do pensamento preocupante relacionado à finitude da vida do idoso, o qual ocasiona angústia para esses indivíduos.

As contribuições da pesquisa provêm dos achados acerca da vulnerabilidade dos cuidadores e ressaltam a necessidade de ações destinadas à prevenção e promoção da saúde dessa população. Frente ao exposto, o estudo demonstrou que a carga horária de cuidados gasta pelos cuidadores e a divisão do cuidado são pontos que devem ser discutidos e planejados com o profissional de saúde, para, desse modo, evitar casos de sobrecarga.

Como limitação do estudo, vale ressaltar que esta pesquisa se deu em um dado centro de atenção a idosos e seus cuidadores, onde ocorrem vários atendimentos envolvendo uma equipe multidisciplinar constituída por enfermeiros, psicólogos, farmacêuticos, médicos e terapeutas ocupacionais, portanto os achados retratam uma realidade particular, que pode divergir de outros cenários e sujeitos, impedindo a generalização dos resultados.

Além disso, pode-se mencionar que, devido à demora de retorno dos cuidadores às consultas subsequentes, a captação foi mediante o contato com os possíveis participantes, solicitando o comparecimento ao setor. Ainda o público que compôs o estudo foi bem heterogênio, esse fato pode ter constituído outra limitação.

\section{CONCLUSÃO}

Depreende-se que a dependência dos idosos com demência e a sobrecarga do cuidado colocam o cuidador em condições de vulnerabilidade biológica e psicológica. Destaca-se que o percentual de cuidadores identificados com níveis preocupantes de sobrecarga foi elevado.

A compreensão da vulnerabilidade do cuidador é o primeiro passo para a construção das prescrições de cuidados que abranjam as necessidades desse público.

De início, a idade elevada dos cuidadores deve ser ponderada como um fator de alerta, pois, nesses casos, naturalmente espera-se que essas pessoas apresentem uma maior vulnerabilidade biológica.

Igualmente, deve-se conhecer o histórico de doenças pregressas do indivíduo no intuito de evitar o agravamento dos sintomas de alguma patologia instalada e prevenir o surgimento de outras. De outra parte, a inserção de outras pessoas para realizar o cuidado é necessária para a qualidade de vida do cuidador.

Além disso, os dados incitam a reflexão acerca do inestimável valor dos Centros de Atenção aos Idosos e seus Cuidadores. Esses locais colaboram com o gerenciamento do cuidado e proporcionam 
uma rede de suporte ao idoso e seu cuidador.

Propõe-se que a enfermagem volte seu olhar para a saúde do cuidador de idosos com demência e considere as consequências desse trabalho e o impacto sobre sua vida. Sob essa conjectura, é necessário identificar os fatores agravantes e atenuantes para, então, traçar estratégias adequadas de intervenções.

Aconselha-se que outros estudos com esse mesmo traço metodológico sejam realizados. Porém, pode ser agregada aos objetivos a comparação entre os resultados dos cuidadores formais e informais com o intuito de identificar possíveis diferenças. Diante disso, recomenda-se, por fim, a realização de estudos longitudinais com os cuidadores, com vistas a obter o controle das variáveis.

\section{REFERÊNCIAS}

1. Lenardt $M H$, Willig $M H$, Seima MD, Pereira LF. A condição de saúde e satisfação com a vida do cuidador familiar de idoso com Alzheimer. Colomb Med. [Internet] 2011;42(Suppl 1) [acesso em 14 dez 2014]. Disponível: http://www.scielo.org.co/pdf/cm/v42n2s1/v42n2s1a3.pdf.

2. Instituto Brasileiro de Geografia e Estatística (IBGE). Síntese de Indicadores Sociais: uma análise das condições de vida da população brasileira. [Internet] Rio de Janeiro: IBGE; 2013 [acesso em 14 dez 2014]. Disponível: https:// biblioteca.ibge.gov.br/visualizacao/livros/liv66777.pdf.

3. World Health Organization (WHO). Dementia Fact Sheets. [Internet] Geneva: WHO; 2013 [acesso em 14 dez 2014]. Disponível: http://www.who.int/medicines/areas/priority_medicines/BP6_11Alzheimer.pdf.

4. Souza EM, da Cunha AP, Melo R, Moreira A. Nurses care for people with dementia: a literature review. J. res.: fundam. care. online. [Internet] 2014;6(3) [acesso em 14 dez 2014]. Disponível: http://dx.doi.org/10.9789/21755361.2014.v6i3.1268-1275.

5. Camacho ACLF, Abreu LTA, Leite BS, Mata ACO, Marinho TF, Valente GSC. An integrative review about nursing care to people with alzheimer's and their caregivers. J. res.: fundam. care. online. [Internet] 2013;5(3) [acesso em 14 dez 2014]. Disponível: http://dx.doi.org/10.9789/2175-5361.2013.v5i3.186-193.

6. Scazufca M. Brazilian version of the Burden Interview scale for the assessment of burden of care in careers of people with mental illnesses. Rev. Bras. Psiquiatr. [Internet] 2002;24(1) [acesso em 19 abr 2016]. Disponível: http:// dx.doi.org/10.1590/S1516-44462002000100006.

7. Cummings JL, Frank JC, Cherry D, Kohatsu ND, Kemp B, Hewett L, et al. Guidelines for managing Alzheimer's disease: part I. Assessment. Am Fam Physician. [Internet] 2002;65(11) [acesso em 19 abr 2016]. Disponível: http:// www.aafp.org/afp/2002/0601/p2263.html.

8. Sidia M, Callegari J. Bioestatística: princípios e aplicações. Porto Alegre: Artmed; 2008.

9. Lago DMSK, Guilhem D, Sousa JA, da Silva KGN, Vieira TS. Physical and psychological burden of caregivers of patients interned in domicile. J Nurs UFPE on line. [Internet] 2015;9(1) [acesso em 19 abr 2016]. Disponível: http:// www.revista.ufpe.br/revistaenfermagem/index.php/revista/article/view/5384/pdf_6999.

10. Lino VTS, Rodrigues NCP, Camacho LAB, O'Dwyer G, de Lima IS, Andrade MKN, et al. Prevalence of overburden in caregivers of dependent elderly and associated factors in a poor area of Rio de Janeiro, Brazil. Cad. Saúde Pública. [Internet] 2016;32(6) [acesso em 01 ago 2016]. Disponível: http://dx.doi.org/10.1590/0102311X00060115.

11. Gaioli CCLO, Furegato ARF, Santos JLF. Perfil de cuidadores de idosos com doença de Alzheimer associado à resiliência. Texto Contexto Enferm. [Internet] 2012;21(1) [acesso em 19 out 2014]. Disponível: http://dx.doi. org/10.1590/S0104-07072012000100017.

12. de Araújo CLO, Oliveira JF, Pereira JM. Perfil de cuidadores de idosos com Doença de Alzheimer. Revista Kairós Gerontologia. [Internet] 2012;15(1) [acesso em 19 out 2014]. Disponível: http://revistas.pucsp.br/index.php/ kairos/article/viewFile/13109/9638.

13. Flores EG, Rivas ER, Seguel FP. Nivel de sobrecarga em el desempeño del rol del cuidador familiar de adulto 
mayor com dependencia severa. Cienc. enferm. [Internet] 2012;18(1) [acesso em 19 out 2014]. Disponível: http:// dx.doi.org/10.4067/S0717-95532012000100004.

14. de Valle-Alonso MJ, Hernández-López IE, Zúñiga-Vargas ML, Martínez-Aguilera P. Sobrecarga y Burnout en cuidadores informales del adulto mayor. Enfermería Universitaria. [Internet] 2015;12(1) [acesso em 19 abr 2016]. Disponível: http://dx.doi.org/10.1016/j.reu.2015.05.004.

15. Valente GSC, Nogueira GA, de Mello LP, Pereira VT, Lindolpho MC, de Sá SPC. Nursing diagnosis for caregivers of elderly with dementia. Rev Enferm UFPE on line. [Internet] 2011;5(8) [acesso em 19 out 2014]. Disponível: http:// www.revista.ufpe.br/revistaenfermagem/index.php/revista/article/viewArticle/1330.

16. Carvalho MFC, Romano-Lieber NS, Bergsten-Mendes G, Secoli SR, Ribeiro E, Lebrão ML, et al. Polypharmacy among the elderly in the city of São Paulo, Brazil - SABE Study. Rev. bras. epidemiol. [Internet] 2012;15(4) [acesso em 19 abr 2016]. Disponível: http://dx.doi.org/10.1590/S1415-790X2012000400013.

17. Garbaccio JL, Garcia TF, Cândida DA. Avaliação da independência de idosos atendidos por uma estratégia de saúde da família. Cogitare Enferm. [Internet] 2013;18(4) [acesso em 19 abr 2016]. Disponível: http://dx.doi. org/10.5380/ce.v18i4.34914. 